\title{
Prediction and evaluation of concealed ore body in 3D environment
}

\author{
ZHU Pengfei ${ }^{1}$, Bai Yun ${ }^{1}, \mathrm{He}_{\text {Zilan }}{ }^{1}$, Kong Weihao ${ }^{1}, \mathrm{Cao} \mathrm{Ke}^{1}$ \\ $\left({ }^{1} \mathrm{CNNC}\right.$ Key Laboratory of Uranium Resources Exploration and Evaluation Technology, Beijing Research Institute of Uranium Geology, \\ Beijing 100029, China)
}

\begin{abstract}
D models of stratigraphy , rock , tectonic , mineralization and so on are established, based on the data of digital uranium exploration achievement, focusing on 3D modeling of uranium deposit 、 evaluation of deep uranium resource. Exploration achievement model are formed by the result above accompany with geophysical geochemical prospecting data and Interpretation. The prediction and evaluation of deep deposits which delineated metallogenic advantageous target area are carried out by weights of evidence method. The applications of digital uranium exploration achievement are realized by the application research above, which improving efficiency and broadening application range and depth of digital exploration achievement.
\end{abstract}

\section{Introduction}

In the 1950s, Allais (Allais M, 1957), Slicher (Slicher LB, 1960), and Harris (Harris DP, 1969) applied statistical methods to the prediction and evaluation of mineral resources, which laid a theoretical foundation for the prediction and evaluation of mineral resources and promoted the evaluation and development of quantitative prediction of mineral resources. In addition, the IGCP 98 project of the International Federation of Geosciences has launched six methods for quantitative prediction and evaluation of mineral resources. The prediction theory and method of mineral statistics are proposed (Zhao Pengda et al., 1994). Singer (1993) proposed the "three-part" resource evaluation method. Wang et al. (1989) studied comprehensive information prediction (Wang Shicheng et al., 1989) and mineral anomaly prediction methods (Zhao et al., 1991). In the 1990s, with the development of GIS technology, the prediction and evaluation of mineral resources entered the GIS era, and a digital prediction and evaluation method system of mineral resources supported by spatial database and GIS spatial analysis technology was formed. Its theories and methods have become the mainstream method of mineral resource prediction and evaluation, and it is widely used in regional mineral resource prospect prediction and evaluation.

With the deepening of mine exploration work, it has been difficult to adapt to the requirements of the development of the times by relying on two-dimensional planes to express and analyze space geological problems. The rapid development of computer graphics technology, $3 \mathrm{D}$ visualization technology, 3D interpolation method and database technology has greatly promoted the development of 3D geology. The combination of threedimensional GIS and three-dimensional geological modeling technology has been applied to convert geological problems from traditional two-dimensional plane to three-dimensional. On the basis of 3D, people use computer 3D modeling technology and geological statistics to estimate reserves to carry out $3 \mathrm{D}$ threedimensional metallogenic prediction of hidden ore bodies, which has become one of the many bright spots in mineral exploration in recent years. Three-dimensional metallogenic prediction research is of great significance to the development of metallogenic prediction theory and the excavation of hidden mines. It greatly expands the thinking space of prospecting and realizes the visualization of deep geological phenomena. It not only provides new prospecting ideas, but also expands the traditional two-dimensional prospecting into a threedimensional prospecting space. It is an extremely important technical method in the quantitative prediction of the location of hidden ore bodies.

\section{Research ideas and technical methods}

The method of quantitative prediction and evaluation of three-dimensional positioning of mineral resources is based on the existing theory of mineralization, theory of mineralization prediction, and the theory of mineral resources exploration and evaluation. By analyzing the background of regional mineralization, the laws of regional mineralization are summarized, and a threedimensional geological model is further established. Through the application of the cube prediction model for prospecting methods and geostatistical methods, we can summarize the prospecting information variables to

Email: zpfjpu@126.com 
construct a prospecting prediction model and delineate the target area. Finally, we carry out the verification and evaluation of the target area, and we realize the integrated management of the original data and provide suggestions for the mine construction. Therefore, geological, geophysical, geochemical, and remote sensing data in the study area were systematically collected. Regarding basic geological data such as comprehensive geological maps, profile maps, and borehole databases, we use geological 3D modeling software to build 3D geological models and 3D surface models, and then carry out special mineral geological surveys for favorable mineralization sites. In addition, we use a three-dimensional mineral prediction model to combine various elements related to mineralization prediction, and we analyze favorable conditions for mineralization, and we look for favorable combined spatial locations. In order to realize the quantitative prediction and evaluation of deep mineral resources, we use three-dimensional technology and virtual reality technology for integrated expression and comprehensive analysis. In general, the entire threedimensional quantitative prediction and evaluation of the ore body is mainly divided into three main modules, which are the establishment of a three-dimensional solid model, visual prediction and evaluation, and integrated analysis of results.

\section{Establishing a 3D solid model}

The establishment of a three-dimensional geological model is the premise and foundation of the threedimensional quantitative prediction and evaluation of ore bodies. We build a three-dimensional geological model of the deposit on the basis of the various two-dimensional plane and profile data collected. The three-dimensional geological model can be divided into surface model, stratum model, structural model, rock mass model, known mineralization characteristic model, and existing geological engineering model. Before modeling, the data we need to collect includes remote sensing image maps, terrain elevation data, geological maps of different scales, measured geological profile maps, mid-plans, borehole catalogue data, exploration line profiles, geophysical interpretation data, and geology at various stages report. In addition, based on plane and section geological data, we combine interpreted geophysical information and rely on GoCAD software's Gaussian discrete interpolation algorithm (DSI), which can make full use of borehole data to build a three-dimensional geological body model. The existing geological research materials are usually paper, Excel, AutoCAD, MapGIS, ArcGIS file types. In order to ensure the comprehensive utilization of data, first of all, we need to classify various data according to their types and their relationships and unify all types of data by converting Data format to define the spatial data structure. Next, we need to further realize the unified storage and management of plane geological maps, geological profile maps, and geophysical and geochemical data.

\section{Visual prediction and evaluation}

The three-dimensional quantitative prediction and evaluation of the ore body is essentially a comprehensive summary of ore-controlling elements, so as to extract a combination of favorable mineralization conditions. The key depends on the geological knowledge of the predicted deposit. The conceptual model of prospecting can effectively guide the quantitative prediction. By establishing quantitative indicators for metallogenic conditions, a digital model for prospecting is constructed. Based on the prospecting digital model and geological model, we extract the favorable elements of metallogenesis and use the prediction theory of geostatistics to realize the three-dimensional metallogenic conditions analysis of deep ore bodies in the study area. In addition, we need to find a favorable combination of metallogenic conditions and quantitatively calculate the amount of resources and reserves, so as to realize the qualitative, quantitative and localized three-dimensional prediction and evaluation of mineral resources in the study area.

The three-dimensional prediction of the ore body is to determine the favorable area of mineralization, thereby optimizing and delineating the target area. The delineation of the target area is mainly based on the spatial distribution of metallogenic favorableness values in the study area, which is an analysis of the metallogenic possibility. We continue to analyze and summarize the three-dimensional geological model, the conceptual model of prospecting, and the digital model of prospecting in the study area, and then we use the three-dimensional modeling software to establish the cube prediction model of the mining area. In addition, according to the quantized three-dimensional cube model, we use statistical methods to statistically analyze the information data contained in the cube unit to calculate the prospecting benefit of each block. Next, we need to count the proportion of each favorable degree range containing known ore blocks and calculate the convergence of each statistical data. Based on the percentage of known ore bodies in the favorableness interval tending to stabilize, the favorableness limit is divided, and then the prospecting target area is determined.

This research mainly uses the three-dimensional evidence weighting method and the three-dimensional prospecting information quantity method for location prediction. Evidence weighting method is a geostatistical method proposed by Agterberg who a Canadian mathematical geologist is. He uses a statistical analysis model, through the superposition and composite analysis of some geoscience information related to mineral formation to make predictions of mineral prospects. Information method is a nonparametric univariate statistical analysis method. The idea is to find out the role of geological factors in guiding the prospecting by studying the distribution of geological factors in all units in the study area. The sum of the information of geological factors in a unit reflects the influence of the unit in mineralization. 


\section{Analysis of achievements}

The work results include a large amount of data and threedimensional models of geological, geophysical, geochemical, and drilling in the study area. We need to explain its further comprehensive integration and application. The integrated analysis of research results is supported by database technology, virtual reality technology and network technology. It uses the spatial database as the management background and integrates the original data, models and results of the research into a coordinate system for unified management analysis. The purpose is to achieve output and three-dimensional display. On the basis of comprehensive analysis of various exploration results and prediction results, we put forward proposals for the exploration and deployment of deep mineral resources. We also put forward the direction of prospecting. According to the delineated target area (predicted results), we need to carry out the verification engineering design in the target area and arrange the borehole verification engineering to determine the location and depth.

\section{[introduction to the author]}

Zhu Pengfei (1978 -), male, senior engineer, works in the Institute of Geology and mineral resources, Beijing Research Institute of Uranium Geology, engaged in the research on 3D geological modeling and resource evaluation.

\section{Reference :}

1. Agterberg F P.Geomathematics:Mathematical Backgroundand Geoscience Applications[M].New York:Elsevier Scien-tific Publishing Company, 1974:1-596.

2. Zhao P D.Theories,principles, and methods for statisticalprediction of mineral deposits[J].Journal of MathematicalGeology,1992(6):589-595.

3. Singer D A.Basic concepts in three-part quantitative assess-ments of undiscovered mineral resources [J].Nonrenewable Resources, 199 3,2(2):69-81.

4. Wang Shiming, Cheng Qiuming. A comprehensive information prospecting model for gold deposits [J]. Journal of Changchun Institute of geology, 1989,19 (3): 311-316

5. Zhao Peng Da, Chi shundu. Preliminary discussion on geological anomalies [J]. Earth Science: Journal of China University of Geosciences, 1991,16 (3): 241248

6. Chen Jianping, Yu Pingping, Shi Rui, et al. Study on $3 \mathrm{D}$ quantitative prediction and evaluation method of regional concealed ore body $[\mathrm{J}]$. Geoscience front, 2014,21 (5): 211-220 